\title{
Un nuevo microbiotérido (Mammalia, Marsupialia, Microbiotheria) de la Formación Pinturas (Mioceno tem- prano) de la provincia de Santa Cruz
}

\author{
Francisco J. GOIN¹, Marcelo F. TEJEDOR², M. Alejandra ABELLO ${ }^{3}$ y Gabriel M. MARTIN²
}

\section{Introducción}

En esta nota se describe una nueva especie de marsupial microbiotérido referible al género Pachybiotherium Ameghino, 1902. La misma está basada en dos especímenes colectados en Enero de 2003 en el noroeste de la provincia de Santa Cruz, durante una expedición paleontológica organizada por el Laboratorio de Investigaciones en Evolución y Biodiversidad (Facultad de Ciencias Naturales, Sede Esquel, Universidad Nacional de la Patagonia). Ambos especímenes fueron exhumados en niveles medios de la Formación Pinturas (Edad-mamífero Santacrucense; Mioceno temprano terminal; Kramarz, 2009; sobre la estratigrafía y edad de la Formación Pinturas, véase Bown y Larriestra, 1990; Fleagle et al., 1995; Kramarz y Bellosi, 2005; Tejedor et al., 2006). Más allá del reconocimiento del nuevo taxón, resulta interesante que en el hipodigma de la nueva especie se incluye un molar superior, el primero hasta ahora conocido para representantes de este género. Este resto permite apreciar características básicas de los dientes superiores de los representantes del género, así como también el conjunto de rasgos que caracterizan el patrón molar de los Microbiotheriidae.

\begin{abstract}
Abreviaturas. IGM, Instituto de Investigaciones en Geociencias, Minería y Química, Museo Geológico Bogotá (INGEOMINAS); MACN, Museo Argentino de Ciencias Naturales "Bernardino Rivadavia", Buenos Aires; MLP, División Paleontología Vertebrados, Museo de La Plata; MPEF-PV, Sección Paleontología Vertebrados, Museo Paleontológico "Egidio Feruglio", Trelew MPM-PV, Museo Regional Provincial "Padre Manuel Jesús Molina", Río Gallegos (Provincia de Santa Cruz), colección
\end{abstract}

1División Paleontología Vertebrados, Museo de La Plata, Paseo del Bosque s/n, 1900 La Plata, Argentina. fgoin@fcnym.unlp.edu.ar ${ }^{2}$ Laboratorio de Investigaciones en Evolución y Biodiversidad (LIEB), Facultad de Ciencias Naturales, Sede Esquel, Universidad Nacional de la Patagonia, Sarmiento 849, 9200 Esquel, Argentina. mtejedor@unpata.edu.ar; gmartin_ar@yahoo.com

32Laboratorio de Sistemática y Biología Evolutiva (LASBE), Museo de La Plata, Paseo del Bosque s/n, 1900 La Plata, Argentina. mabello@fcnym.unlp.edu.ar

CAsociación Paleontológica Argentina
Paleontología Vertebrados. M1, M2, M3, M4, molares superiores; $\mathrm{m} 1, \mathrm{~m} 2, \mathrm{~m} 3, \mathrm{~m} 4$, molares inferiores. La nomenclatura dentaria sigue a Goin et al. (2003). Todas las medidas son en $\mathrm{mm}$.

\section{Paleontología sistemática}

Supercohorte MARSUPIALIA Illiger, 1811

Orden Microbiotheria Ameghino, 1889

Familia MicrobiotHERIIDAE Ameghino, 1887

Género Pachybiotherium Ameghino, 1902

Especie tipo. Pachybiotherium acclinum Ameghino, 1902 (véase Marshall, 1982: fig. 28a-c y la Fig. 2 de este trabajo).

Especies incluidas. P. acclinum, P. minor Goin, 1997 (véase Goin, 1997: figs. 11.1. A-B y 11.2.A-B) y la nueva especie aquí descripta.

Distribución. Mioceno temprano-medio, América del Sur.

\section{Pachybiotherium illuminatum, sp. nov.} (figuras 3.1-5, 4)

Etimología. Del latín illuminatus, iluminar; en referencia al nuevo conocimiento aportado por el molar superior referido a la nueva especie.

Tipo. MPM-PV 1805, un fragmento mandibular derecho con alvéolos de m1-2 y los m3-4 completos (Figs. 3.1-4, 4).

Hipodigma. El tipo y MPM-PV 1806, un M3 derecho (Figs. 3.5, 4).

Distribución. Portezuelo Sumich Sur, Estancia Los Toldos, a unos $80 \mathrm{~km}$ al sur de Perito Moreno, Departamento Lago Buenos Aires, Provincia de Santa Cruz, Argentina (figura 1). Niveles medios de la Formación Pinturas (véase Kramarz y Bellosi, 2005: fig. 2); Mioceno temprano terminal (Edad-mamífero Santacrucense).

Medidas. Altura mandibular por debajo del m3: 11,2; ancho mandibular por debajo del m4: 11,3; largo m3-4: 6,3; largo m3: 3,4; ancho m3 (trigónido): 2,1; ancho m3 (talónido): 2,2; largo M3: 3,4; ancho M3: 3,8 (véase también la tabla 1).

Diagnosis. Se diferencia de las otras dos especies del género por su mayor tamaño, la mayor reducción del $\mathrm{m} 4$, el largo proporcionalmente mayor del talónido

AMGHB2-0002-7014/10\$00.00+.50 


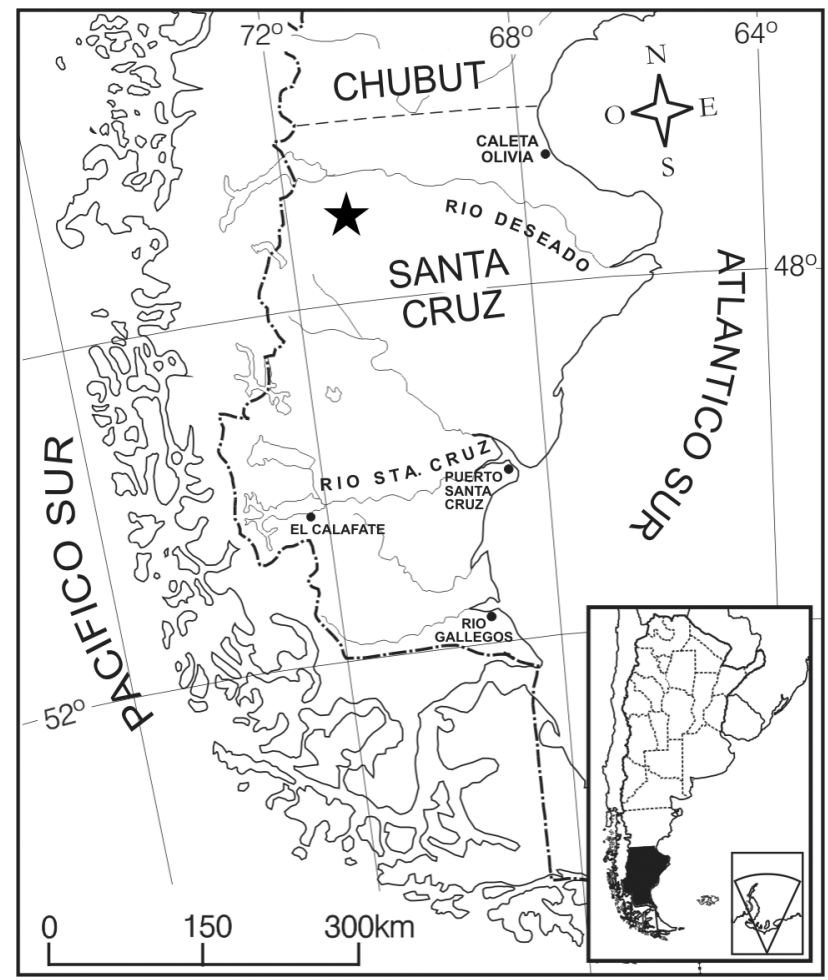

Figura 1. Mapa de la provincia de Santa Cruz (República Argentina) indicando la localidad fosilífera Portezuelo Sumich Sur (estrella) / map of Santa Cruz Province (Argentina) indicating the Portezuelo Sumich Sur fossil locality (star).

del m3, la mayor reducción del paracónido en el m3 y la posición más retrasada del metacónido con relación al protocónido en los m3-4; se diferencia de $P$. acclinum en que, en los molares inferiores, el hipoconúlido está más separado del entocónido, el entocónido es proporcionalmente más grande y el ápice del protocónido es de posición más vertical (i.e., no está inclinado hacia atrás); se diferencia de $P$. minor en que los molares inferiores tienen talónidos proporcionalmente más anchos y largos, y en que el cíngulo anterobasal está más desarrollado.

Descripción. El cuerpo mandibular es alto y robusto (figuras 3.1-3), con la rama horizontal sin una aparente inflexión medial. La cresta coronoidea (sensu Wible, 2003) es ancha y robusta y se origina en un punto medio de la rama horizontal, debajo de los m2$\mathrm{m} 3$, formando un ángulo de aproximadamente $130^{\circ}$ con respecto a esta rama. La fosa masetérica es profunda y su parte anterior está bien marcada. La mandíbula presenta los cuatro alvéolos de los molares anteriores (m1-2), de tamaño subigual a los del m3. El tipo ha preservado los m3-4, los cuales muestran un leve desgaste. Tanto en el $\mathrm{m} 3$ como en el $\mathrm{m} 4$ se aprecia en el trigónido cierta retracción del metacónido respecto del protocónido. El paracónido del m3 está muy reducido y, al igual que el del $\mathrm{m} 4$, está algo comprimido en sentido anteroposterior. Por detrás y algo labialmente a la cúspide del paracónido se aprecia una muy pequeña cúspide o rugosidad de homología incierta, mucho más marcada en el m3 que en el $\mathrm{m} 4$. Tanto en el m3 como en el m4 la muesca de la paracrístida es amplia. El protocónido es robusto y de implantación vertical, desde la base hasta el ápice. El cíngulo anterobasal está apenas desarrollado, aunque más que en $P$. minor. El talónido del m3 es subigual en ancho al trigónido; por el contrario, en el m4 el talónido es más angosto. En el m3 se aprecia con claridad un bien desarrollado entocónido, siendo el hipocónido poco saliente y el hipoconúlido proporcionalmente grande y bien separado del entocónido ocupando una posición media sobre la cara posterior del talónido. El trigónido del $\mathrm{m} 4$ es muy similar al del m3, aunque algo más angosto y con el metacóni-

Tabla 1. Medidas de los molares inferiores de las distintas especies del género Pachybiotherium.. Las medidas relativas al ancho de los molares corresponden al ancho del trigónido. Las medidas correspondientes a Pachybiotherium minor fueron tomadas de Goin (1997: tabla 11.1). L, largo; A, ancho. Todas las medidas son en mm / Measurements of the lower molars of species of the genus Pachybiotherium. Measurements relative to molar width correspond to trigonid width. Measurements of Pachybiotherium minor were taken from Goin (1997: table 11.1). L, length; A, width. All measurements are in $\mathrm{mm}$.

\begin{tabular}{|c|c|c|c|c|c|c|c|c|c|}
\hline Especie & Ejemplar & Lm1 & Am1 & Lm2 & Am2 & Lm3 & Am3 & Lm4 & Am4 \\
\hline P. illuminatum sp. nov & MPM-PV 1805 (tipo) & --- & --- & --- & -- & 3,4 & 2,1 & 2,93 & 1,77 \\
\hline P. minor & IGM 253027 & -- & -- & -- & -- & --- & 1,10 & --- & -- \\
\hline P. minor & IGM 253026 & --- & --- & --- & --- & --- & 1 & --- & --- \\
\hline P. minor & IGM 253051 (tipo) & --- & --- & --- & -- & --- & --- & 2,15 & 1,10 \\
\hline P. acclinum & MACN 52-370 (tipo) & 3,29 & 1,84 & --- & --- & 3,24 & 1,88 & --- & -- \\
\hline P. acclinum & MPEF-PV 551 & 3,48 & 1,99 & --- & --- & --- & --- & --- & -- \\
\hline P. acclinum & MLP 92-X-10-10 & 2,84 & 1,51 & 3,22 & 1,77 & 3,21 & 1,88 & --- & --- \\
\hline P. acclinum & MLP 82-V-2-111 & 3,10 & 1,63 & 3,36 & 2,02 & 3,26 & 1,84 & --- & --- \\
\hline
\end{tabular}



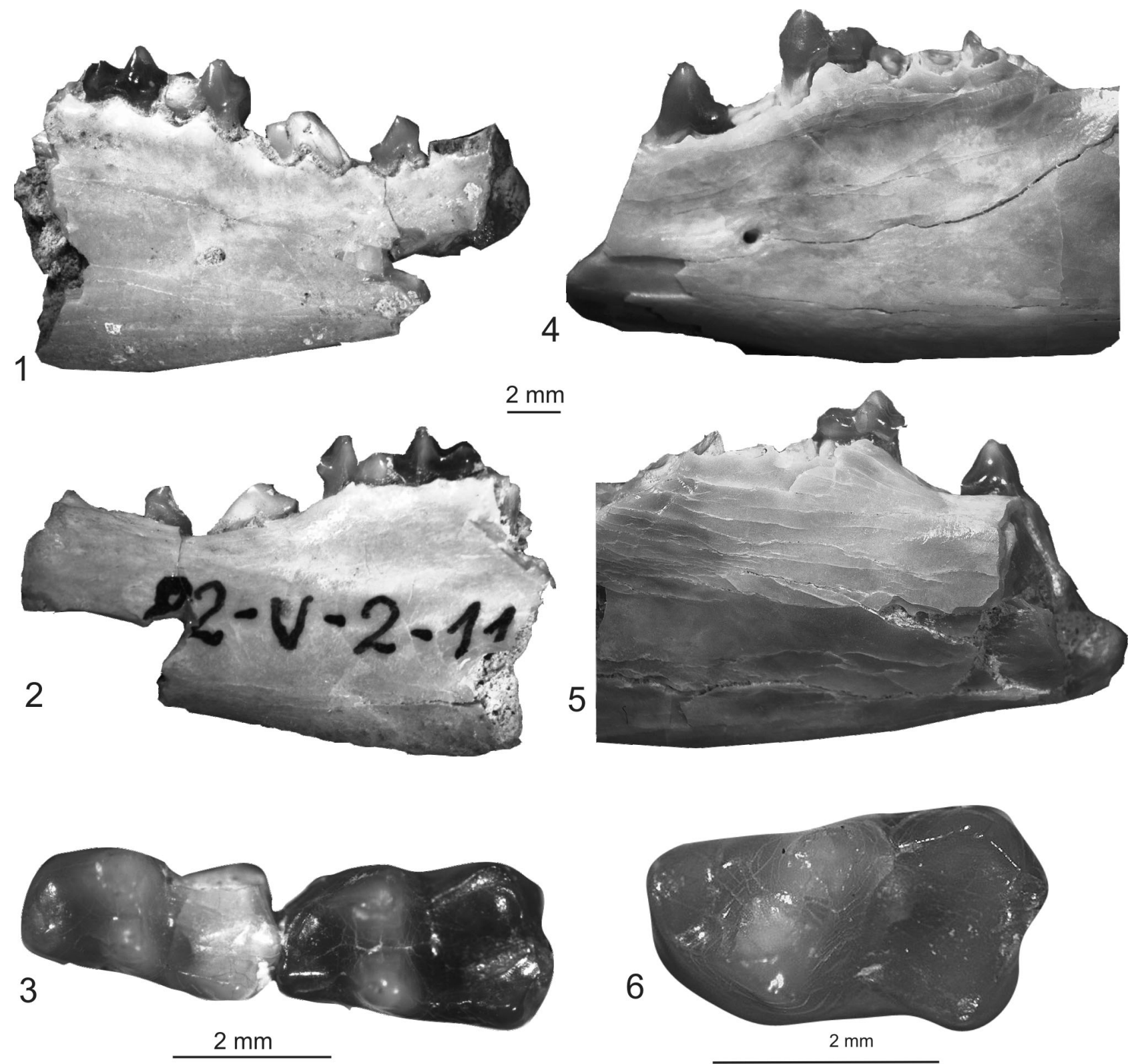

Figura 2. Pachybiotherium acclinum Ameghino. 1-3, MLP 82-V-2-111, fragmento de dentario derecho con los p2-m2 en vistas labial (1) y lingual (2) y detalle de los m1-2 en vista oclusal (3) / fragment of right dentary with $p 2-m 2$ in labial (1) and lingual (2) views, and a detail of m1-2 in occlusal view (3). 4-6, MPEF-PV 551, fragmento de dentario izquierdo con los p3-m1 en vistas labial (4), lingual (5) y detalle del $\mathrm{m} 1$ en vista oclusal (6) / fragment of left dentary with $p 3-m 1$ in labial (4) and lingual (5) views, and a detail of $m 1$ in occlusal view (6).

do aún más desplazado posteriormente; el talónido, por su parte, muestra el entocónido y el hipocónido reducidos (en el m4 el entocónido no está desarrollado), siendo el hipoconúlido más grande y terminando en punta hacia atrás. En este diente, el hipocónido se ubica en línea con el protocónido, estando el área del entocónido completamente reducida. El molar superior es aquí referido a un M3. El protocono es ancho, el para y metacono son subiguales en altura (figura 4) y la plataforma estilar está muy reducida. La preparacrista, muy corta y orientada casi paralela al eje dentario, está alineada con la centrocrista, la cual es recta. Un par de minúsculas cúspides en el centro de la plataforma estilar podrían corresponder a una StC subdividida. La postmetacrista, si bien es muy corta comparada con la de otros microbiotéridos, es más larga que la preparacrista y se orienta en forma más oblicua respecto del eje longitudinal del diente. A pesar del desgaste, la estructura de la corona en la base lingual del metacono indica que existió un metacónulo de desarrollo moderado. El diente carece de ectoflexo. La preprotocrista parece formar un cíngulo anterior que se une con la esquina parastilar del diente, aunque esto podría ser efecto del desgaste. 

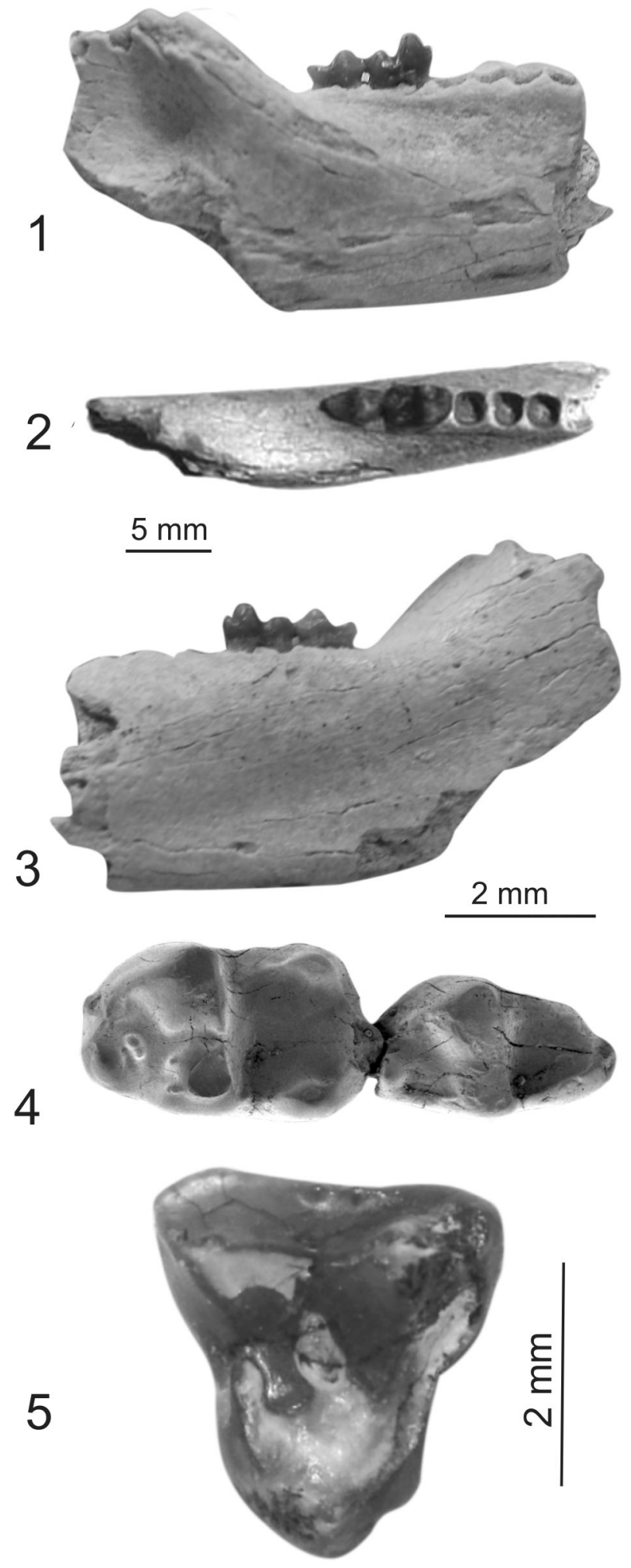

Figura 3. Pachybiotherium illuminatum sp. nov. 1-3, MPM-PV 1805 (tipo), un dentario derecho con los m3-4 en vistas labial (1), oclusal (2) y lingual (3) / (type), a right dentary with m3-4; labial (1), occlusal (2) and lingual (3) views; 4, detalle en vista oclusal de los m3-4 del ejemplar tipo / detail in occlusal view of the m3-4 of the type specimen; 5, MPM-PV 1806, M3 derecho en vista oclusal. / right M3 in occlusal view.

AMEGHINIANA 47 (1), 2010

\section{Comentarios}

En trabajos previos se argumentó sobre la pertenencia de Pachybiotherium a los Microbiotheria (Goin, 1991; para una revisión de la posición sistemática y rasgos diagnósticos del género véase Goin, 1997). La nueva especie aquí descripta puede ser referida a Pachybiotherium en base a la presencia, en su ejemplar tipo, de los siguientes caracteres diagnósticos: (1) gran altura de la rama mandibular; (2) molares inferiores con trigónidos estrechos, (3) cíngulos anterobasales pobremente desarrollados; (4) paracónidos muy reducidos; (5), metacónidos levemente retrasados respecto de los protocónidos; (6) hipoconúlidos levemente separados de los entocónidos y (7) m4 levemente reducido respecto del $\mathrm{m} 3$.

Clemens (1966) sugirió varios criterios que permiten reconstruir series dentarias a partir de piezas aisladas. A los efectos del presente análisis, sólo es posible realizar comparaciones sobre la base de uno de ellos: los tamaños relativos. Según el mismo, es posible referir a P. illuminatum el ejemplar MPM-PV 1806 (un M3 aislado). Las estimaciones llevadas a cabo por Clemens (1966) para los cocientes de las longitudes $\mathrm{M} 1 / \mathrm{m} 1, \mathrm{M} 2 / \mathrm{m} 2$ y M3/m3 arrojan valores entre 0,91 y 1,04 para tres especies de didélfidos vivientes estudiadas por este autor como marco de referencia. El cociente $\mathrm{M} 3 / \mathrm{m} 3$ equivale a 1,0 para el ejemplar aquí referido a P. illuminatum, valor que cae dentro de este rango. Otro criterio adicional a los aquí mencionados es el de confrontar las estructuras oclusales de los molares supuestamente antagonistas; por ejemplo, el ancho del protocono del molar superior en relación al ancho del talónido del inferior, o las longitudes de las crestas funcionalmente antagónicas en la oclusión. En la figura 4 se aprecia una vista labial del M3 (MPM-PV 1806) y de los m3-4 (MPM-PV 1805, el tipo) de P. illuminatum en situación de oclusión. Puede verse que las longitudes de la paracrístida del $\mathrm{m} 4 \mathrm{y}$ de la postmetacrista del M3 son muy similares, así como también las de los otros dos pares de crestas que, en oclusión, constituyen antagonistas funcionales: la postparacrista y la premetacrista en el molar superior y la crístida oblicua y la posthipocrístida en el inferior. En síntesis, se verifica una ajustada geometría ocusal en los molares funcionalmente antagónicos, lo que apoya las dos hipótesis aquí formuladas sobre el ejemplar MPM-PV 1806: (1) que es referible a la nueva especie P. illuminatum y (2) que es un M3.

El M3 de P. illuminatum muestra los rasgos característicos de los microbiotéridos neógenos (e.g., Microbiotherium): notable reducción de la plataforma estilar, preparacrista corta y orientada casi paralelamente al eje dentario, ausencia de cúspides estilares reconocibles (Goin et al., 2007a). La mayor diferencia entre $P$. illuminatum y otros microbiotéridos neóge- 
nos es la persistencia, en los molares superiores, de un metacónulo de desarrollo moderado. Otros microbiotéridos (e.g., Microbiotherium) también muestran un metacónulo, pero éste es comparativamente más reducido. En otros rasgos, sin embargo, $P$. illuminatum muestra algunas especializaciones: ausencia de ectoflexo y muy corta postmetacrista del M3.

Pachybiotherium illuminatum es hasta el momento la única de las especies del género entre cuyos restos se cuentan molares superiores. Goin (1997) incluyó tentativamente a un molar superior (IGM 253029) entre los materiales referibles a P. minor. Los argumentos para dicha asignación fueron tres: centrocrista recta, fusión del paracónido con la StB y su tamaño, esperable para un M1 de dicha especie. Sin embargo, estos rasgos también son característicos de los molares deciduos de numerosos didélfidos. Por otra parte, el tamaño del protocono de este ejemplar es relativamente reducido, lo cual contradice su pertenencia a los Microbiotheriidae. Por esta razón optamos aquí por descartar al ejemplar IGM 253029 como perteneciente a $P$. minor.

Los únicos microbiotéridos vivientes se restringen a la especie Dromiciops gliroides Thomas, de comportamiento arborícola, hábitos nocturnos y dieta predominantemente faunívora (i.e., compuesta por invertebrados, aunque con una mayor proporción de artrópodos) y secundariamente frugívora (Mann, 1955; Pearson, 1983; Amico y Aizen, 2000). Por su parte, las especies de Pachybiotherium son los representantes de mayor tamaño de la familia Microbiotheriidae. Se destacan por presentar un gran desarrollo vertical del dentario por debajo de la serie molar, un rasgo compartido con numerosos grupos de primates de hábitos alimentarios frugívoros (Vinyard et al., 2007 y bibliografía allí citada), así como también con los extintos marsupiales Caroloameghiniidae y Polydolopimorphia, de hábitos inferidos similares (Goin, 2006). Los molares inferiores de las especies de este género presentan talónidos anchos en los molares inferiores y robustos protoconos en los superiores, lo que indica que la función de "mortero" de estas estructuras antagónicas era relativamente importante durante el cierre oclusal. Adicionalmente, las estructuras de corte (paracrístida, postmetacrista) son proporcionalmente reducidas. Estos rasgos sugieren la hipótesis de que los representantes del género eran de hábitos alimentarios esencialmente frugívoros.

Una revisión reciente sobre la significación climática de las paleofloras australes de América del Sur señala el desarrollo, hacia los $34^{\circ} \mathrm{S}$, de una "Flora Subtropical Neógena" en coincidencia con el óptimo climático del Mioceno (ca. 15-17 Ma), rango temporal en el que se habrían depositado los sedimentos de la localidad de procedencia de P. illuminatum aquí estu-

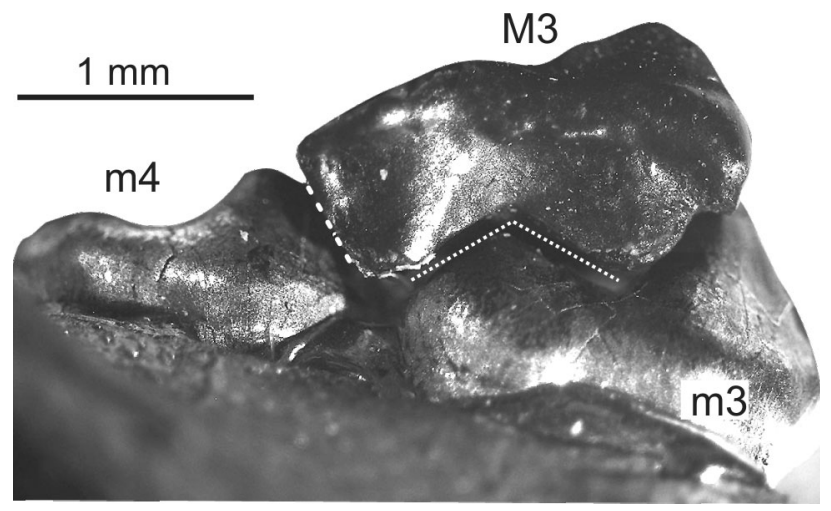

Figura 4. Pachybiotherium illuminatum sp. nov. Molar superior (MPM-PV 1806, M3) e inferiores (MPM-PV 1805, m3-4) en oclusión. La línea punteada indica la orientación de la centrocrista del M3 en relación con las estructuras de corte del antagonista oclusal (m3); la línea quebrada hace lo propio con la postmetacrista del M3 y la paracrístida del m4. / upper and lower molars (MPM-PV 1806, M3, and MPM-PV 1805, m3-4) in occlusion. The dot line indicates the M3 centrocrista's orientation as opposed to the occlusal antagonist (m3); the dashed line indicates the relation between the postmetacrista of M3 and paracristid of $m 4$.

diada (vide supra). Esta flora habría estado caracterizada por la dominancia de elementos neotropicales y endémicos, además de la persistencia de elementos austral-antárticos, australasianos y pantropicales (Hinojosa, 2005). Es difícil precisar si este tipo de flora ocurrió también en la latitud de Sumich Sur (y de las localidades pinturenses en general) hacia esta época. Sin embargo, una amplia representación de elementos neo- y pantropicales podría explicar una elevada proporción de frugívoros en esta asociación faunística respecto de las asociaciones subsecuentes (e.g., las de la Edad-mamífero Santacrucense). Hinojosa (2005) hace extensiva la "Flora Subtropical Neógena" al Mioceno temprano (ca. 20 Ma), i.e., en simultáneo con el desarrollo de la fauna de Edad Colhuehuapense. En esta última es, precisamente, donde ocurre el primer registro del género Pachybiotherium (P. acclinum Ameghino, 1902), además del de un probable Caluromyidae (Didelphimorphia), formas cuyos representantes vivientes son mayoritariamente frugívoros (Goin et al., 2007b). Es interesante señalar que la tercera especie conocida de este género, P. minor Goin, 1997, ocupa un rango geográfico netamente tropical (La Venta, Colombia; Edad Laventense). Podría postularse, a título de hipótesis, que las especies de Pachybiotherium constituyen indicadores de climas cálidos.

\section{Agradecimientos}

Al personal del Museo Regional Padre Manuel Jesús Molina de Río Gallegos por el apoyo ofrecido para el desarrollo de nues- 
tros proyectos en Santa Cruz y a M. Molina, propietario de la estancia Los Toldos, por permitirnos trabajar en su propiedad. A S. Vincon y C. Etcheverri por su colaboración en los trabajos de campo. A A. Candela, a un revisor anónimo y a S. Bargo, del Comité Editor, por las valiosas sugerencias realizadas. F.J.G. agradece al CONICET (PIP 5621) y a la Fundación Alexander von Humboldt. Los trabajos de campo fueron financiados por la L.S.B. Leakey Foundation a M.F.T.

\section{Bibliografía}

Ameghino, F. 1902 (1902-1904). Première contribution à la connaissance de la faune mammalogique des couches à Colpodon. Boletín de la Academia Nacional de Ciencias (Córdoba) 17: 71-141.

Amico, G. y Aizen, M.A. 2000. Mistletoe seed dispersal by a marsupial. Nature 408: 929-930.

Bown, T.M. y Larriestra, C.N. 1990. Sedimentary paleoenvironments of fossil platyrrhine localities, Miocene Pinturas Formation, Santa Cruz Province, Argentina. Journal of Human Evolution 19: 87-119.

Clemens, W.A. 1966. Fossil mammals of the Type Lance Formation, Wyoming. Part II. Marsupialia. University of California Publications in Geological Sciences 62: 1-122.

Fleagle, J.G. 1990. New fossil platyrrhines from the Pinturas Formation, Southern Argentina. Journal of Human Evolution 19: 61-85.

Fleagle, J.G., Powers, D.W., Conroy, G.C. y Watters, J.P. 1987. New fossil platyrrhines from Santa Cruz Province, Argentina. Folia Primatologica 48: 65-77.

Fleagle, J.G., Bown, T.M., Swisher, C. y Buckley, G. 1995. Age of the Pinturas and Santa Cruz Formations. $6^{\circ}$ Congreso Argentino de Paleontología y Bioestratigrafía (Trelew), Actas: 129-135.

Goin, F.J. 1991. [Los Didelphoidea (Mammalia, Marsupialia) del Cenozoico Tardío de la región pampeana. Tesis Doctoral Facultad de Ciencias Naturales y Museo de la Universidad Nacional de La Plata., 323 pp. Inédito.].

Goin, F.J. 1997. New clues for understanding Neogene Marsupial radiations. En: R.F. Kay, R.H. Madden, R.L. Cifelli y J. Flynn (eds.), A History of the Neotropical Fauna. Vertebrate Paleobiology of the Miocene in Colombia. Smithsonian Institution Press, Washington D. C., pp. 185-204.

Goin, F.J. 2006. A review of the Caroloameghiniidae, Paleogene South American "primate-like" marsupials (?Didelphimorphia, Peradectoidea). En: D. Kalthoff, T. Martin y T. Möors (eds.), Festband für Herrn Professor Wighart $v$. Koenigswald anlässlich seines 65. Geburtstages. Palaeontographica Abt. A 278. E. Schweizerbart'sche Verlagsbuchhandlung, Stuttgart, pp. 57-67.

Goin, F.J., Candela, A.M. y Muizon, C. de. 2003. The affinities of Roberthoffstetteria nationalgeographica (Marsupialia) and the origin of the Polydolopine molar pattern. Journal of Vertebrate Paleontology 23: 869-876.

Goin, F.J., Zimicz, N., Reguero, M.A., Santillana, S.N., Marenssi, S.A. y Moly, J.J. 2007a. New marsupial (Mammalia) from the Eocene of Antartica, and the origins and affinities of Microbiotheria. Revista de la Asociación Geológica Argentina 62: $597-603$
Goin, F. J., A. Abello, E. Bellosi, R. Kay, R. Madden y A. A. Carlini. 2007b. Los Metatheria sudamericanos de comienzos del Neógeno (Mioceno temprano, Edad-mamífero Colhuehuapense). Parte 1: Introducción, Didelphimorphia y Sparassodonta. Ameghiniana 44: 29-71.

Hinojosa, L.F. 2005. Cambios climáticos y vegetacionales inferidos a partir de paleofloras cenozoicas del sur de Sudamérica. Revista Geológica de Chile 32: 95-115.

Kramarz, A.G. 2009. Adiciones al conocimiento de Astrapothericulus (Mammalia, Astrapotheria): anatomía cráneo-dentaria, diversidad y distribución. Revista Brasileira de Paleontología 12: 55-66.

Kramarz, A.G. y Bellosi, E.S. 2005. Hystricognath rodents from the Pinturas Formation, Early-Middle Miocene of Patagonia, biostratigraphic and paleoenvironmental implications. Journal of South American Earth Sciences 18: 199-212.

Mann, G. 1955. Monito del Monte. Dromiciops australis Philippi. Investigaciones Zoológicas Chilenas 2: 159-166.

Marshall, L.G. 1982. Systematics of the South American marsupial family Microbiotheriidae. Fieldiana, Geology (New Series) 10: 175.

Pearson, O.P. 1983. Characteristics of a mammalian fauna from forests in Patagonia, southern Argentina. Journal of Mammalogy 64: 476-492.

Vinyard, C.J., Ravosa, M.J., Williams, S.H., Wall, C.E., Johnson, K.R. y Hylander, W.L. 2007. Jaw-Muscle Function and the Origin of Primates. En: M.J. Ravosa y M. Dagosto (eds.), Primate origins: adaptations and evolution. Springer, New York, pp. 179-231.

Tejedor, M.F. 2002. Primate canines from the early Miocene Pinturas Formation, southern Argentina. Journal of Human Evolution 43: 127-141.

Tejedor, M.F. 2005a. New specimens of Soriacebus adrianae, with comments on pitheciin primates from the Miocene of Patagonia. Ameghiniana 42: 249-251.

Tejedor, M.F. 2005b. New fossil platyrrhine from Argentina. Folia Primatologica 76: 146-150.

Tejedor, M.F., Tauber, A.A., Rosenberger, A.L., Swisher III, C.C. y Palacios, M.E. 2006. New primate genus from the Miocene of Argentina. Proceedings of the National Academy of Sciences, U.S.A. 103: 5437-5441.

Thomas, O. 1894. On Micoureus griseus, Desm., with the Description of a new Genus and Species of Didelphyidae. Annals and Magazine of Natural History 6: 184-188.

Wible, J.R. 2003. On the cranial osteology of the short-tailed opossum Monodelphis brevicaudata (Didelphidae, Marsupialia). Annals of Carnegie Museum 72: 137-202.
Recibido:

Aceptado: 